\title{
The Hector Survey: integral field spectroscopy of 100,000 galaxies
}

\author{
J. Bland-Hawthorn \\ Sydney Institute for Astronomy (SIfA), School of Physics, The University of Sydney, NSW \\ 2006, Australia \\ email: jbh@physics.usyd.edu.au
}

\begin{abstract}
In March 2013, the Sydney-AAO Multi-object Integral field spectrograph (SAMI) began a major survey of 3400 galaxies at the AAT, the largest of its kind to date. At the time of writing, over a third of the targets have been observed and the scientific impact has been immediate. The Manga galaxy survey has now started at the SDSS telescope and will target an even larger sample of nearby galaxies. In Australia, the community is now gearing up to deliver a major new facility called Hector that will allow integral field spectroscopy of 100 galaxies observed simultaneously. By the close of the decade, it will be possible to obtain integral field spectroscopy of 100,000 galaxies over 3000 square degrees of sky down to $r=17$ (median). Many of these objects will have HI imaging from the new ASKAP radio surveys. We discuss the motivation for such a survey and the use of new cosmological simulations that are properly matched to the integral field observations. The Hector survey will open up a new and unique parameter space for galaxy evolution studies.
\end{abstract}

Keywords. galaxies: evolution - galaxies: kinematics and dynamics - galaxies: structure techniques: imaging spectroscopy

\section{Introduction}

A chain of argument is only as strong as its weakest link. In this respect, our understanding of how galaxies evolve from the first seeds to the present day is seriously incomplete. We have a reasonable picture of how the first dark matter structures came together out of the initial matter perturbations. But just how gas settled into these structures to form the first stars and subsequent generations remains an extremely difficult problem. From an observational perspective, the main advances in evolutionary studies have come from spatially resolved imaging and spectroscopy across many wavebands, from the development of optical and imaging spectroscopy since the 1960s, the HI and radio continuum surveys of the 1970s, improvements in infrared imaging and spectroscopy in the 1980s, and the emergence of mid-infrared, UV, x-ray and gamma-ray satellites in the 1990s to the present day. These advances have come from a combination of detailed studies of individual objects and large surveys of global parameters.

Over the past twenty years, imaging surveys from the Hubble Space Telescope (far field) and the Sloan Digital Sky Survey (near field) have been particularly effective in identifying evolution of galaxy parameters with cosmic time and with environment across large-scale structure. This has been matched by large galaxy surveys using multi-object spectroscopy (MOS), most notably at the AAT, SDSS, VLT, Magellan and Keck telescopes (e.g. York et al. 2000; Colless et al. 2001; Driver et al. 2011). MOS instruments provide a single spectrum within a fixed fibre aperture at the centre of each galaxy; spatial information must be drawn from multi-wavelength broadband images. While this approach has its place, the question always remained as to whether imaging spectroscopy was feasible for a cosmologically significant sample. 

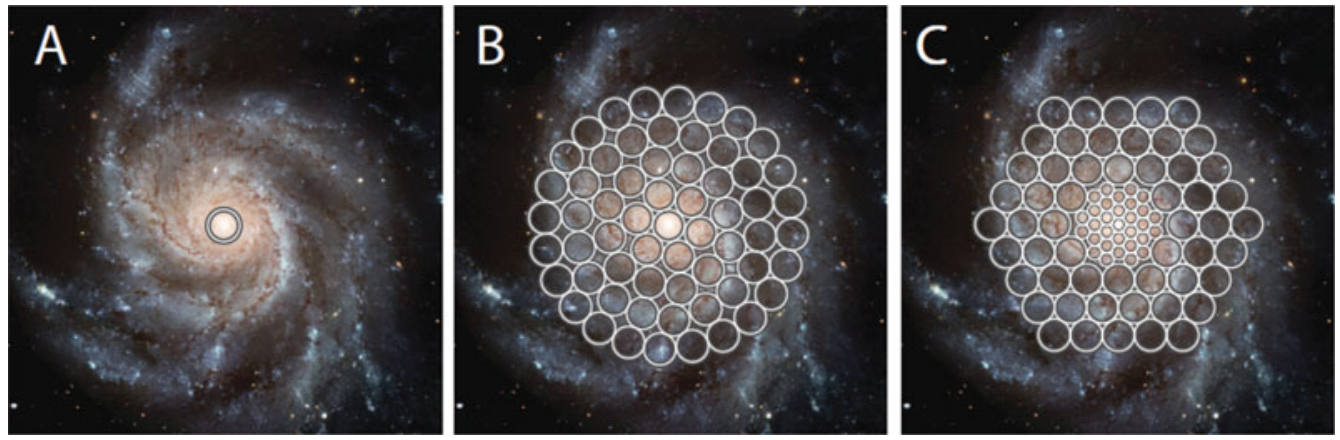

Figure 1. (a) Single fibre apertures are the basis for galaxy surveys to date; (b) the 61-fibre configuration of the SAMI hexabundle; (c) the 85-fibre (54 large, 31 small) configuration of the Hector hexabundle under development at the University of Sydney.

In the 1980s, the scientific potential for imaging spectroscopy was clear from early Fabry-Perot (Bland et al. 1987; Cecil 1988; Amram et al. 1992) and tuneable filter studies (Jones \& Bland-Hawthorn 2001). After the pioneering work of Courtès et al.(1988), integral field spectroscopy (IFS) soon exploited the plunging costs of large-area detectors to dominate extragalactic studies today (Hill 2014). Recent integral field surveys involving hundreds of nearby galaxies include ATLAS-3D (Cappellari et al. 2011) and CALIFA (Sánchez et al. 2012). A useful summary of recent scientific results is given by Glazebrook (2013).

So how do we combine the power of the MOS and IFS techniques, the 'missing link' in astronomical instrumentation? VLT KMOS provides an elegant solution involving small image slicers: this instrument employs 24 configurable arms that position pickoff mirrors at specified locations in the Nasmyth focal plane. But this expensive technology would be prohibitively costly to adapt for the degree-scale fields of cosmologically motivated MOS instruments. Seven years ago, we began to look at compact fibre bundles (hexabundles) that would work with existing robotic positioners (Bland-Hawthorn et al. 2011; Bryant et al. 2011). This led to the Sydney-AAO Multi-object Integral field spectrograph (SAMI), the first instrument of its kind. SAMI deploys 13 IFUs, each with a field of view of $15^{\prime \prime}$ across a 1-degree patrol field (Croom et al. 2012). Each IFU consists of a bundle of 61 optical fibres lightly fused to have a high $(\sim 75 \%)$ filling factor (Bryant et al. 2014a). SAMI is installed on the 3.9-m Anglo-Australian Telescope (AAT), feeding the existing AAOmega spectrograph. For the first time, SAMI allows very large samples of IFS observations to be obtained in a short period of time.

\section{The SAMI Galaxy Survey: early results}

The SAMI Galaxy Survey is an ongoing project to obtain integral field spectroscopic data for $\sim 3400$ galaxies, with an expected completion date of mid 2016. The bundles are dithered with respect to sky to wash out the footprint (Fig. 1(b)). An example of the data quality is shown in Fig. 2. At the time of writing, roughly 1000 galaxies have been observed, including the pilot survey of $\sim 100$ galaxies. The updated instrument performance, target selection, survey parameters and reduction procedures are discussed elsewhere (Bryant et al. 2014b; Sharp et al. 2014). The Galaxy Survey, which includes both cluster and field objects selected from the GAMA survey, had an early data release in 2014 (Allen et al. 2014). The use of GAMA fields means that the SAMI data are complemented by many surveys, including GALEX MIS, VST KiDS, VISTA VIKING, 


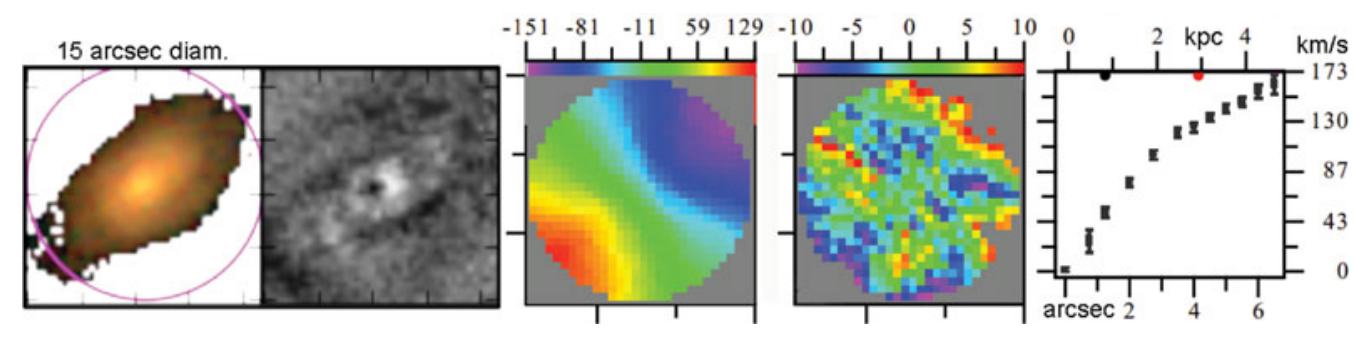

Figure 2. SAMI results on a nearby galaxy: (a) red stellar continuum; (b) residual after disk model subtracted to reveal spiral arms; (c) gas velocity field in $\mathrm{km} \mathrm{s}^{-1}$; (d) $<7 \%$ residuals after rotation model subtracted; (e) gas rotation as a function of radius (with errors shown). SAMI also provides stellar velocities and emission-line ratios giving information on gas chemistry and ionization conditions. The circular bundle in (a) is dithered using small shifts on sky to remove the footprint (Fig. 1) and fill out the field with complete data.

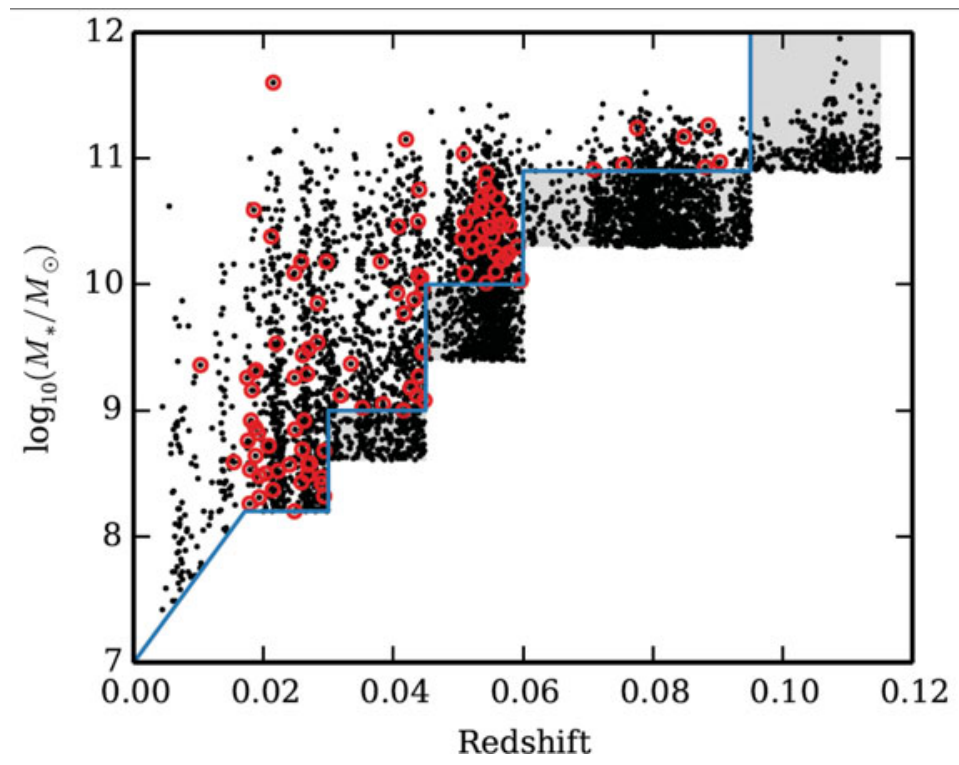

Figure 3. Stellar mass and redshift for all galaxies in the GAMA field regions of the SAMI Galaxy Survey (black points); the early data release sample are also shown (red circles). The blue boundaries indicate the primary selection criteria, while the shaded regions indicate lower-priority targets. Large-scale structure within the GAMA regions is seen in the overdensities of galaxies at particular redshifts (Allen et al. 2014).

WISE, Herschel-ATLAS, GMRT and ASKAP (Driver et al. 2011). Early science results include studies of galactic winds (Fogarty et al. 2012; Ho et al. 2014), a universal dynamical relation for all galaxy types (Cortese et al. 2014), the kinematic morphology-density relation for early-type galaxies (Fogarty et al. 2014), bar streaming in spiral galaxies (Cecil et al. 2014), and star formation in dwarf galaxies (Richards et al. 2014); see the related contributions in this volume.

\section{Hector instrument}

The main limitation of SAMI is the need to plug each bundle into pre-cut metal plates. A. Bauer has made a short movie - www.youtube.com/watch?v=MB5H-5XZ9ZE - of this labour intensive process, something we would rather avoid with 100,000 targets in 
mind. The Hector concept (Lawrence et al.2012) will exploit the full two-degree field of the AAT prime focus using robotically positioned hexabundles. The AAO has invested several decades in robotic technology for positioning fibres over large focal planes. Their most recent development is the semi-autonomous "starbug" that positions fibres (or bundles) with a piezo-electric tube that walks over a glass plate. For a movie of how this works in practice, see here: www.youtube.com/watch?v=YvZDF54Si5w. Recent tests by S. Richards and C. Betters show that this technology is ideal for moving the bundles around. An update on the performance statistics for starbugs is given in Goodwin et al. (2010).

Much like HETDEX and VLT MUSE before us, we will need to replicate cheap spectrographs to handle 8500 fibres from the 100 hexabundles. Replicating spectrographs at $R=2000$ is relatively inexpensive using 4000-pixel Andor detectors, for example. We are exploring simple designs involving curved VPH gratings but it is unclear if this technology can reach our goal of $R=\lambda / \delta \lambda \approx 4000$ across the optical band (370-900 nm). A resolution and rough costings should be ready by early 2015 . The modular design of Hector means that the first devices will be ready for use by the end of the SAMI Galaxy Survey in mid 2016.

The need for a new hexabundle with a supersampled inner bundle (Fig. 1(c)) is to minimise loss of spatial resolution over the core regions of galaxies. Essentially all SAMI galaxies are brightest over the inner scale length where the gravitational potential is changing most rapidly. The benefits of proper sampling includes more accurate modelling of the inner potential (e.g. bars), sensitivity to kinematic substructure, higher order moments, and so forth (Naab et al. 2013). The new design allows for excellent sampling within an effective radius $\left(R_{e}\right)$ for most galaxies while allowing us to reach $2 R_{e}$ with the larger outer fibre apertures. This design also has the added benefit of providing better intrinsic sampling of more distant and compact targets.

\section{Hector science}

\subsection{Big questions}

Fossil signatures. The formation history of galaxies leaves its imprint on the gas and stellar kinematic properties of present day galaxies. Epochs dominated by gas dissipation will result in the formation of flattened stellar distributions (disks), supported by rotation. During merger dominated phases the stellar systems experience stripping and violent relaxation, existing cold gas might be driven to the central regions causing starbursts, trigger the formation and growth of supermassive black holes or be expelled form the systems in a galactic wind. This, in turn, will impact the distribution of cold gas and the kinematics of forming stars.

Using cosmological 'zoom' simulations of representative synthetic galaxies, Naab et al. (2013) demonstrate that gas dissipation and merging result in observable features (at the present day) in the $2 \mathrm{D}$ kinematic properties of galaxies, which are clear signatures of distinct formation processes. Dissipation favours the formation of fast rotating systems and line-of-sight velocity distributions with steep leading wings, a property that can be directly traced back to the orbital composition of the systems (Röttgers et al. 2014; Sharma et al. 2012). Merging and accretion can result in fast or slowly rotating systems with counter-rotating cores, cold nuclear or extended (sometimes counter-rotating) disks showing dumpbell-like features - all observed in real galaxies. The strength of these kinematic signatures will be influenced by feedback from supernovae and AGN, but also by the mass of the galaxies and their environment. 


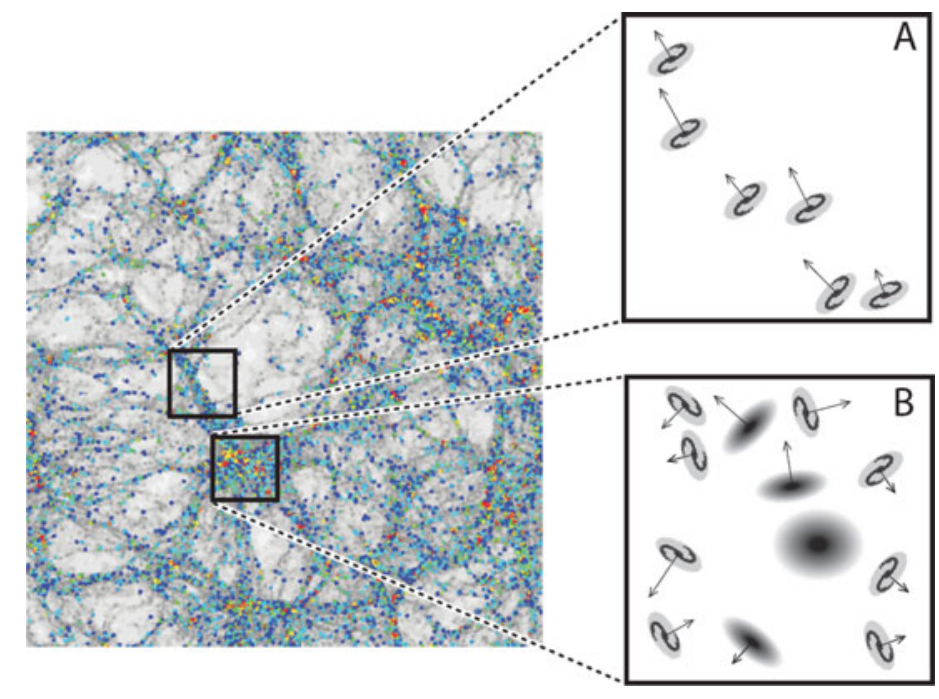

Figure 4. Cosmological simulation (GIF consortium) of the universe today within a $30 \mathrm{Mpc}$ volume. Blue, yellow and red dots are young, middle-aged and old galaxies; the grey is unprocessed gas. The most detailed simulations predict that (A) low-mass galaxy spins align along filaments, and (B) spins are more randomised in dense regions (e.g Dubois et al.2014).

Low mass star forming disk galaxies favor low-density environments, predominantly grown by accretion of gas and subsequent in-situ star formation, and are affected by stellar feedback. Higher mass early-type galaxies form in high-density environments - possibly affected by feedback from accreting super-massive black holes - and their late assembly involves merging with other galaxies, which might also be of an early type. Before now, it was not possible to perform a statistically meaningful comparison of kinematic properties of galaxy populations to observed population properties, like the observed increasing fraction of slow versus fast rotators for early-type galaxies as a function of environmental density (Cappellari et al. 2012). With the new Eagle simulations (Schaye et al. 2014), we can tackle these questions for the first time using the large simulated volume and the higher spatial resolution.

With the Hector instrument, the 2D kinematic (gas, stars) and abundance patterns will be compared over different environs. Preliminary work will be possible with SAMI and Manga, but the higher target density across large-scale structure will allow a more detailed statistical connection to investigate trends with environment. We will attempt to (i) identify characteristic formation histories, (ii) identify properties of progenitor galaxy populations, (iii) assess the impact of the major feedback mechanisms (from massive stars and AGN) on the kinematic properties of high and low mass galaxies. This study that will be supported by higher resolution cosmological zoom simulations for characteristic cases.

The origin of angular momentum. An interesting future area of study is the build-up of angular momentum in galaxies and across different environs (Fig. 4). Codis et al. (2012) exploit the Horizon simulations $(z>1.3)$ and find an interesting interplay between the gas and dark matter during accretion onto galaxies. The gas and dark matter phases separate when sheets and filaments form between expanding voids of different sizes. Material flows out of voids at a rate that depends on the size of the void. The dark matter collapses to form asymmetric sheet or filament but the gas does not. The gas 


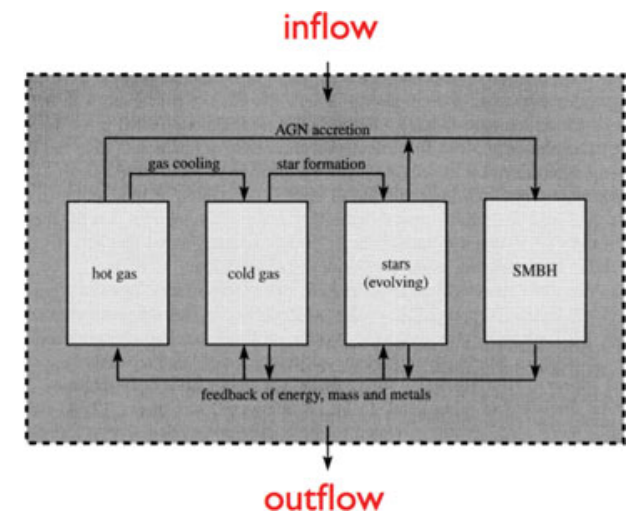

Figure 5. A schematic of the inner workings of a galaxy (adapted from Mo et al. 2010), akin to a 4 -stroke engine fuelled by gas with a complex exhaust system. Galaxy evolution is an extremely complex process that demands much larger 3D spectroscopic surveys and the measurement of new parameters.

undergoes shocks and compresses to form a medium whose main concentration is offset from the dark matter the protocloud. The protocloud oscillates slowly in the gravity field of the collapsed dark matter sheet and rains onto galaxies as a cold or a warm flow. This idea has far-reaching consequences. The wider the separation of gas and dark matter, the longer the delay in accreting to the outer disk leading to galaxies with different spin properties and star formation histories. The Horizon team find that low mass gas-accreting galaxies develop spins aligned with filaments (Fig. 4) whereas the high mass galaxies are anti-aligned due to major collisions Dubois et al. (2014). The signal is expected in the angular momentum vector field (spin direction and magnitude) when averaged over many thousands of galaxies.

To see this weak signature at $z \sim 0.1$, we need to observe of order 60,000 galaxies in a contiguous volume (Trowland et al. 2013) although a larger sample may be required (Dubois et al. 2014). This requires mass and rotation estimates for stars and/or gas in each galaxy, which Hector is uniquely situated to do. In this paradigm, the collective properties of galaxies in sheets and filaments may be different in character across the hierarchy. Once again, galaxies within a single filament, for example, are expected to have correlated properties but the properties can vary between different filaments. If this is correct, it will radically overhaul our ideas on how galaxies form and evolve because now the disk properties of galaxies are linked to the large-scale environment. In the earlier classical paradigm, shock heating removes any vestige of where the gas came from, thereby removing any large-scale correlations; the hot gas simply cools and collapses to a disk within the halo (Fall \& Efstathiou 1980).

\subsection{Matched data from GAMA and HI surveys}

The GAMA survey is the ideal input catalogue for the SAMI survey because of its supporting data. As we have shown, galaxies are observed in volume-limited regions at several different redshifts (Fig. 3) with a Petrosian limit of about $r \approx 17-17.5$ for the faintest sources. The GAMA field selection means that our survey data are complemented by many surveys from UV to radio wavelengths (Driver et al. 2011). Ideally, for the Hector survey, we would require the GAMA survey to be extended about a magnitude deeper and over a larger region of sky. By the end of the decade, the Large Synoptic Survey Telescope (LSST) will provide very deep multiband photometry across the Southern Sky. 
The GAMA depth and selection complements the all-sky HI surveys soon to be carried out by ASKAP in Australia (Duffy et al. 2012). Some fraction of the envisaged Hector sources will have spatially resolved (10" FWHM) HI imaging and spectroscopy over roughly $\sim 1^{\prime}$ diameter. There are to be two major HI surveys: (1) the Widefield ASKAP L-band Legacy All-sky Blind surveY (WALLABY) is a shallow $3 \pi$ sr survey $(z<0.26)$ which will survey the mass and dynamics of more than $6 \times 10^{5}$ galaxies; (2) a deeper small-area HI survey, called Deep Investigation of Neutral Gas Origins (DINGO), will trace the evolution of $\mathrm{HI}(z<0.43)$ over a cosmological volume of $4 \times 10^{7} \mathrm{Mpc}^{3}$ detecting up to $10^{5}$ galaxies.

\subsection{Survey design}

All large surveys, both stellar and extragalactic, struggle to defend the survey size with any statistical rigour. This is especially true for galaxy evolution studies since many free parameters are needed to capture the microphysics in galaxies (Fig. 5) and the relative importance of different microphysics may vary with the underlying density field and the galaxy's mass.

Our initial study indicates that $10^{5}$ galaxies over a contiguous volume is of order the size we need to probe (i) variations on local overdensity; (ii) different redshifts; (iii) a range of galaxy masses at each redshift; (iv) galaxy orientations; (v) sufficient cell division in the galaxy colour-magnitude diagram. We also need sufficient numbers of objects per bin to counteract complexity and fine structure in galaxies. A key uncertainty is the association of galaxies with local overdensity. Statistical 'crowding' techniques (e.g. five nearest neighbours) are intrinsically uncertain for measuring local density and indeed there are many methods on offer (Muldrew et al. 2012). The target density needs to be sufficiently high, as for the SAMI Galaxy Survey, to ensure groups and clusters are well sampled (Bryant et al. 2014b).

To examine survey parameters more qualitatively needs high-resolution cosmological simulations where individual galaxy properties are resolved. The necessary simulations are only now becoming available for the first time (e.g. Schaye et al. 2014). A proper survey estimate depends ultimately on what is being measured. Broad categories like fast vs. slow rotators may require smaller samples whereas weak measurables (e.g. GaussHermite parameters $\left.h_{3}, h_{4}\right)$, or detailed properties defined by many parameters, push us to larger samples.

We have begun a study of the Eagle simulations through synthetic IFS observations of the stars and gas in each evolving galaxy (cf. Naab et al. 2013). There are basic properties that we would like to have, e.g. fractional variations in slow rotators with respect to fast rotators as a function of environment. Fogarty et al. (2014) have shown that, in the SAMI survey, the ratio of slow to fast rotators may vary across cluster environments. Importantly, Naab et al. (2013) argue the case for good spatial sampling to measure high order moments $\left(h_{3}, h_{4}\right)$ of the stellar kinematics which may also vary in interesting ways over large-scale structure due to preserved kinematic subtructure.

In summary, the Hector survey will allow us to explore how galaxy properties vary across a contiguous volume of the universe. The huge database will provide information on galaxy properties, in particular, stellar and gas kinematics, the presence of nuclear activity (starburst vs. black hole), the star formation distribution, the spread of chemical abundance in both stars and gas, and so forth. The Vienna conference has underscored the broad community support for the the SAMI and Manga surveys which in turn build on the early successes of the ATLAS-3D and CALIFA surveys. Future galaxy studies will be dominated by IFS observations of cosmologically significant numbers of galaxies supported by large HI surveys. 


\section{Acknowledgements}

JBH acknowledges an ARC Federation Fellowship and LIEF grant for the SAMI instrument and an ARC Australian Laureate Fellowship for the ongoing development of the Hector instrument. JBH acknowledges insights from members of the SAMI team, including S. Croom, J. Bryant, J. Allen, L. Fogarty, S. Richards and R. Sharp. We are indebted to J. Lawrence, the Anglo-Australian Observatory and the Anglo-Australian Telescope for their role in realising the SAMI project. My thanks to G. Cecil for producing Fig. 2 and to T. Naab for insights on where the EAGLE simulations can take us.

\section{References}

Allen, J. T., et al. 2014, MNRAS submitted, arXiv:1407.6068 Amram, P., Le Coarer, E., Marcelin, M., et al. 1992, A\&\&A Suppl., 94, 175 Bland, J., Taylor, K., \& Atherton, P. D. 1987, MNRAS, 228, 595

Bland-Hawthorn, J., Bryant, J., Robertson, G., et al. 2011, Optics Express, 19, 2649

Bryant, J. J., et al. 2011, MNRAS, 415, 2173

Bryant, J. J., et al. 2014a, MNRAS, 438, 869

Bryant, J. J., Owers, M. S., Robotham, A. S. G., et al. 2014b, arXiv:1407.7335

Cappellari, M., et al. 2011, MNRAS, 416, 1680

Cappellari, M., McDermid, R. M., Alatalo, K., et al. 2012, Nature, 484, 485

Cecil, G. 1988, ApJ, 329, 38

Cecil, G., Fogarty, L., Bland-Hawthorn, J., et al. 2014, ApJ, submitted

Codis, S., Pichon, C., Devriendt, J., et al. 2012, MNRAS, 427, 3320

Colless, M., Dalton, G., Maddox, S., et al. 2001, MNRAS, 328, 1039

Cortese, L., Fogarty, L., Ho, I.-T., et al. 2014, ApJ, submitted

Croom, S. M., et al. 2012, MNRAS, 421, 872

Driver, S. P., et al. 2011, MNRAS, 413, 971

Dubois, Y., Pichon, C., Welker, C., et al. 2014, MNRAS, 444, 1453

Duffy, A. R., Meyer, M. J., Staveley-Smith, L., et al. 2012, MNRAS, 426, 3385

Fall, S. M. \& Efstathiou, G. 1980, MNRAS, 193, 189

Fogarty, L. M. R., et al. 2012, ApJ, 761, 169

Fogarty, L. M. R., et al. 2014, MNRAS, 443, 485

Glazebrook, K. 2013, PASA, 30, 56

Goodwin, M., Heijmans, J., Saunders, I., et al. 2010, SPIE, 7739

Hill, G. J. 2014, Advanced Optical Technologies, 3, 265

Ho, I., Kewley, L. J., Dopita, M. A., et al. 2014, arXiv:1407.2411

Jones, D. H. \& Bland-Hawthorn, J. 2001, ApJ, 550, 593

Lawrence, J., Bland-Hawthorn, J., Bryant, J., et al. 2012, SPIE, 8446

Mo, H., van den Bosch, F. C., \& White, S. 2010, Galaxy Formation and Evolution, CUP

Muldrew, S. I., Croton, D. J., Skibba, R. A., et al. 2012, MNRAS, 419, 2670

Naab, T., Oser, L., Emsellem, E., et al. 2013, arXiv:1311.0284

Richards, S. N., Schaefer, A. L., Lopez-Sanchez, A. R., et al. 2014, arXiv:1409.4495

Röttgers, B., Naab, T., \& Oser, L. 2014, arXiv:1406.6696

Sánchez S. F., et al. 2012, A\&\&A, 538, A8

Schaye, J., Crain, R. A., Bower, R. G., et al. 2014, arXiv:1407.7040

Sharma, S., Steinmetz, M., \& Bland-Hawthorn, J. 2012, ApJ, 750, 107

Sharp R., et al. 2014, MNRAS submitted, arXiv:1407.5237

Trowland, H. E., Lewis, G. F., \& Bland-Hawthorn, J. 2013, ApJ, 762, 72

York D. G., et al. 2000, AJ, 120, 1579 